\title{
Spin imbalance and spin-charge separation in a mesoscopic superconductor
}

\author{
C. H. L. Quay ${ }^{1 \star}$, D. Chevallier ${ }^{1}$, C. Bena ${ }^{1,2}$ and M. Aprili ${ }^{1,3}$
}

\begin{abstract}
What happens to spin-polarized electrons when they enter a superconductor? Superconductors at equilibrium and at finite temperature contain both paired particles (of opposite spin) in the condensate phase as well as unpaired, spin-randomized quasiparticles. Injecting spin-polarized electrons into a superconductor (and removing pairs) thus creates both spin and charge imbalances ${ }^{1-7}$, which must relax when the injection stops, but not necessarily over the same time (or length) scale. These different relaxation times can be probed by creating a dynamic equilibrium between continuous injection and relaxation; this leads to constant-in-time spin and charge imbalances, which scale with their respective relaxation times and with the injection current. Whereas charge imbalances in superconductors have been studied in great detail both theoretically ${ }^{8}$ and experimentally ${ }^{9}$, spin imbalances have not received much experimental attention ${ }^{6,10,11}$ despite intriguing theoretical predictions of spin-charge separation effects ${ }^{12,13}$. Here we present evidence for an almost-chargeless spin imbalance in a mesoscopic superconductor.
\end{abstract}

A pure spin imbalance in a superconductor can be understood in the following manner: imagine injecting spin-randomized electrons continuously into a small superconducting volume and taking out Cooper pairs. The number of electron-like quasiparticles increases, that is, their chemical potential $\mu_{\mathrm{QP}}$ rises whereas that of the Cooper pairs $\mu_{\mathrm{P}}$ drops by the same amount to conserve particle number. This charge imbalance was first observed in a pioneering experiment, where $\mu_{\mathrm{QP}}-\mu_{\mathrm{P}}$ was measured ${ }^{1,2,14}$. (Hereafter $\mu_{\mathrm{P}} \equiv 0$, that is, all chemical potentials are measured with respect to that of the condensate.) If the injected electrons are (or become) spin-polarized, in general $\mu_{\mathrm{QP} \uparrow} \neq \mu_{\mathrm{QP} \downarrow} \neq \mu_{\mathrm{P}}$, we can define a charge imbalance $\mu_{\mathrm{C}} \equiv\left(\mu_{\mathrm{QP} \uparrow}+\mu_{\mathrm{QP} \downarrow}\right) / 2$ and spin imbalance $\mu_{\mathrm{S}} \equiv\left(\mu_{\mathrm{QP} \uparrow}-\mu_{\mathrm{QP} \downarrow}\right) / 2$ (ref. 13). If charge relaxes faster than spin, a situation may arise in which $\mu_{\mathrm{C}}=0$ while $\mu_{\mathrm{S}} \neq 0$. This is our chargeless spin imbalance. (See Supplementary Information for more details.) In the experiment, $\mu_{\mathrm{QP} \uparrow}-\mu_{\mathrm{P}}$ and $\mu_{\mathrm{QP} \downarrow}-\mu_{\mathrm{P}}$ are measured as a voltage drop between a spin-sensitive electrode and the superconductor.

We implement a mesoscopic version of an experiment proposed in refs 12,13; this offers two practical advantages: the detector can be placed within a spin relaxation length $\lambda_{\mathrm{S}}$ of the injection point and all out-of-equilibrium signals are enhanced by the small injection volume. In diffusive transport, $\lambda_{\mathrm{S}}=\left(D \tau_{\mathrm{S} 2}\right)^{1 / 2}$, where $\tau_{\mathrm{S} 2}$ is the spin relaxation time and $D$ the diffusion constant $\left(\sim 5 \times 10^{-3} \mathrm{~m}^{2} \mathrm{~s}^{-1}\right.$ in our samples $\left.{ }^{15}\right)$. Our samples are FISIF lateral spin valves ${ }^{16}$, where the $\mathrm{F}$ are ferromagnets (Co), the $\mathrm{I}$ are insulators $\left(\mathrm{Al}_{2} \mathrm{O}_{3}\right)$ and $\mathrm{S}$ is the superconductor $(\mathrm{Al})$, as shown in Fig. 1a. The SIF junctions have sheet resistances of $\sim 1.6 \times 10^{-6} \Omega \mathrm{cm}^{2}$ (corresponding to a barrier transparency $T \sim 5 \times 10^{-5}$ ) and tunnelling is the main transport mechanism through the insulator. By sweeping an external magnetic field parallel to the ferromagnetic electrodes, F1 and F2, we can align or anti-align their magnetizations because of their different magnetic shape anisotropies (Fig. 1c). We simultaneously perform local and non-local transport measurements using standard lock-in techniques at low temperature $(70 \mathrm{mK}-4 \mathrm{~K})$ : we apply a current across the junction $\mathrm{J} 1$, between $\mathrm{F} 1$ and $\mathrm{S}$, so that spin-polarized electrons are injected into the superconductor, and we measure the voltage drops and differential resistances across J1 ('local', between F1 and S) and across J2 ('non-local', between F2 and S; Fig. 1b). The distance between J1 and J2 varies between $200 \mathrm{~nm}$ and $500 \mathrm{~nm}$, within the $\mathrm{Al}$ spin relaxation length ${ }^{16}$. The non-local voltage drop at $\mathrm{J} 2$ is proportional to either $\mu_{\mathrm{QP} \uparrow}-\mu_{\mathrm{P}}$ or $\mu_{\mathrm{QP} \downarrow}-\mu_{\mathrm{P}}$, depending on the relative alignments of F1, F2 and the magnetic field. (See ref. 17 and Supplementary Information.)

We first measure the nonlocal magneto-resistance at $4 \mathrm{~K}$, with the aluminium in its normal (non-superconducting) state, to identify the switching fields of the ferromagnets (Fig. 1c). The amplitude of the nonlocal magneto-resistance signal as a function of the distance between the ferromagnetic electrodes (in different samples) also allows us to extract the spin relaxation length in the normal state of $\mathrm{Al}$, assuming an exponential spatial decay (Fig. 1c, inset) as expected in diffusive metallic spin valves ${ }^{16}$. This yields $\lambda_{\mathrm{S}}=450 \pm 50 \mathrm{~nm}, \tau_{\mathrm{S} 2}=40 \pm 10 \mathrm{ps}$ and a spin polarization of $P_{\mathrm{Co}} \sim$ $10 \%$. All these results are consistent with previous experiments ${ }^{16,18}$. In particular, the low Co polarization often observed in highly transparent planar tunnel junctions such as ours ${ }^{19}$ is due to the barrier strength dependence of the relative contributions of the $s$ and $d$ bands to the tunnelling current ${ }^{20}$.

At the base temperature of our dilution refrigerator $(70 \mathrm{mK})$, at which the $\mathrm{Al}$ is superconducting (we measure $T_{\mathrm{C}} \sim 1.23 \mathrm{~K}$ ), we first study our device with the ferromagnets aligned with each other and with the magnetic field. Figure $2 \mathrm{a}$ shows the non-local differential resistance across $\mathrm{J} 2$ as a function of the voltage across J1. Between 0 and $1,500 \mathrm{G}$, we notice a doublepeak structure (that is, a dip then a peak), which intensifies with increasing magnetic field, on a relatively field-independent smooth background.

We show below that this double-peak structure results from spin accumulation in $\mathrm{S}$ whereas the background results from charge imbalance. We treat spin and charge imbalances independently as, to a first approximation, they are not coupled.

\footnotetext{
${ }^{1}$ Laboratoire de Physique des Solides (CNRS UMR 8502), Bâtiment 510, Université Paris-Sud, 91405 Orsay, France, ${ }^{2}$ Institute de Physique Théorique, CEA Saclay 91190 Gif-sur-Yvette, France, ${ }^{3}$ Kavli Institute at Cornell for Nanoscale Science, Cornell University, Ithaca, New York 14853, USA. *e-mail: charis.quay@u-psud.fr.
} 
a
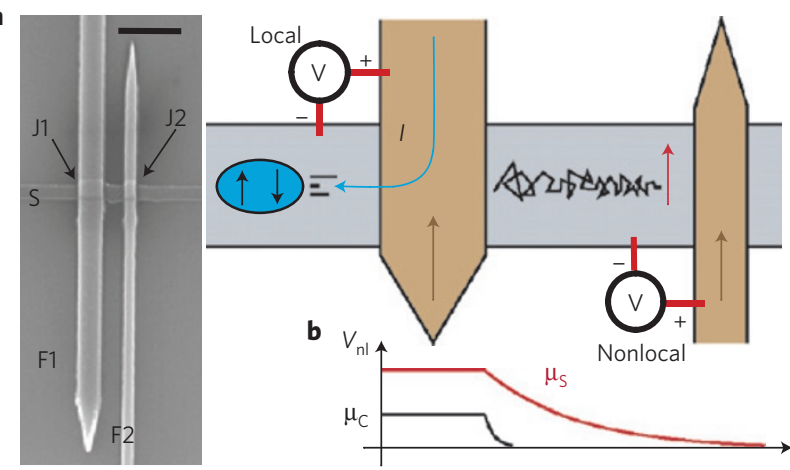

c

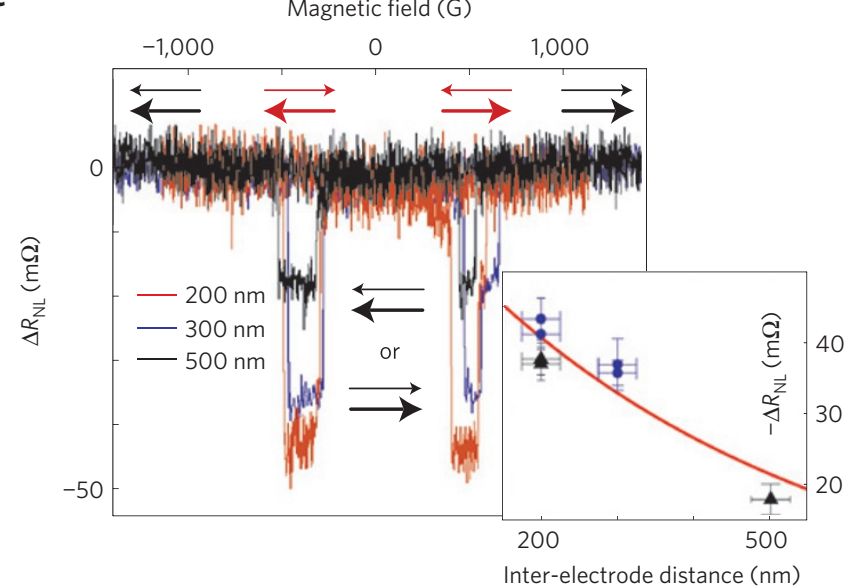

Figure 1 | Device characterization and measurement set-up. a, Scanning electron micrograph of a typical device (8B3, scale bar, $1 \mu \mathrm{m})$. b, Schematic drawing of the same and of the measurement set-up. A current $I$ is injected from a ferromagnet ( $\mathrm{F} 1, \mathrm{Co} / \mathrm{Pd})$ into a superconductor $(\mathrm{S}, \mathrm{Al})$ through a tunnel barrier. The nonlocal voltage $V_{\mathrm{NL}}$ and nonlocal differential resistance $R_{\mathrm{NL}}=\mathrm{d} V_{\mathrm{NL}} / \mathrm{d} /$ are measured between a distant ferromagnetic electrode ( $F 2, \mathrm{Co} / \mathrm{Pd}$ ) and $\mathrm{S}$ as a function of the magnetic field (applied parallel to F1 and F2) and temperature; this probes the chemical potential of the spin up or down electrons with respect to the Cooper pairs, depending on the relative orientations of F1, F2 and the magnetic field. The local voltage and local differential resistance $R=\mathrm{dV} / \mathrm{d} /$ (between $\mathrm{S}$ and F1) are measured simultaneously. The schematic drawing also illustrates a spin imbalance which survives longer in time and over a longer distance than the associated charge imbalance. c, Nonlocal magnetoresistance measurements at $4 \mathrm{~K}$ (where the aluminium is in its normal state) allow us to identify the relative alignments of F1 and F2 (Inset). The dependence of the magnetoresistance signal on device length (distance between $\mathrm{F} 1$ and F2) yields a spin flip length of $450 \pm 50 \mathrm{~nm}$ and a spin relaxation time $\tau_{\mathrm{sf}}=48 \pm 10 \mathrm{ps}$. Data from devices represented by black triangles have been normalized to account for a larger Al width ( $300 \mathrm{~nm}$ instead of $200 \mathrm{~nm}$, see equations in main text).

We first discuss spin imbalance. In F1, spin-up and spindown electrons have densities of states (DOS) $n_{\uparrow}$ and $n_{\downarrow}$, constant within the energy range of interest. (The polarization is $P=\left(n_{\uparrow}-n_{\downarrow}\right) /\left(n_{\uparrow}+n_{\downarrow}\right)$ and the total DOS $\left.N_{\mathrm{F}}=n_{\uparrow}+n_{\downarrow}.\right)$ In S, as has been observed in tunnelling spectroscopy experiments ${ }^{21,22}$, an external in-plane magnetic field splits the quasiparticle Bardeen-Cooper-Schrieffer (BCS) DOS through the Zeeman effect: $n_{\mathrm{QP} \downarrow(\uparrow)}(E)=\left(E \pm \mu_{\mathrm{B}} H\right) /\left(\left(E \pm \mu_{\mathrm{B}} H\right)^{2}-\Delta^{2}\right)^{1 / 2}$. In our experiment, this BCS DOS is also smoothed out through orbital pair-breaking, which provides a depairing mechanism for the Cooper pairs ${ }^{23,24}$. (See Supplementary Information for details on the causes and effects of pair-breaking in our experiment; we use the measured DOS in our calculations and fits to data.) At equilibrium, the

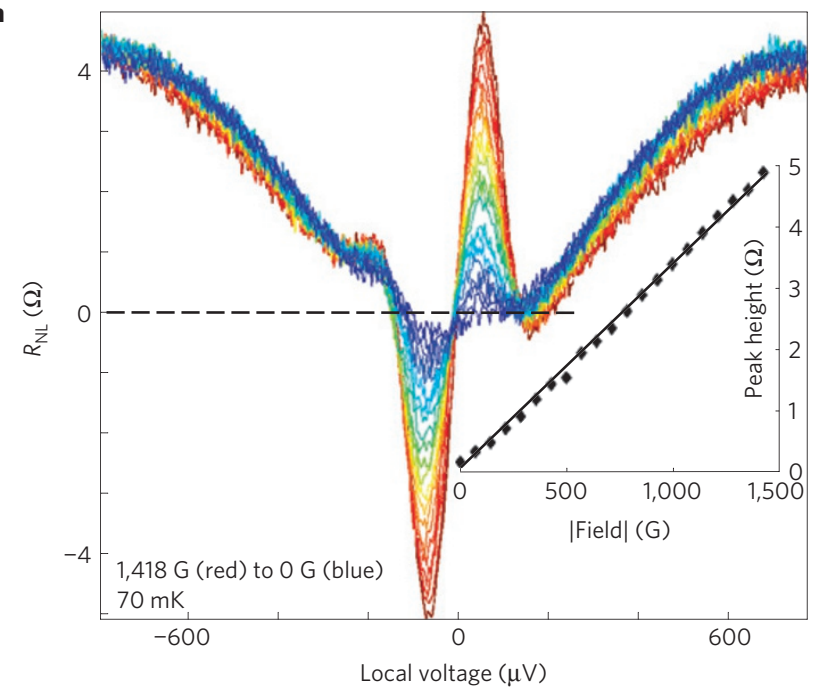

b
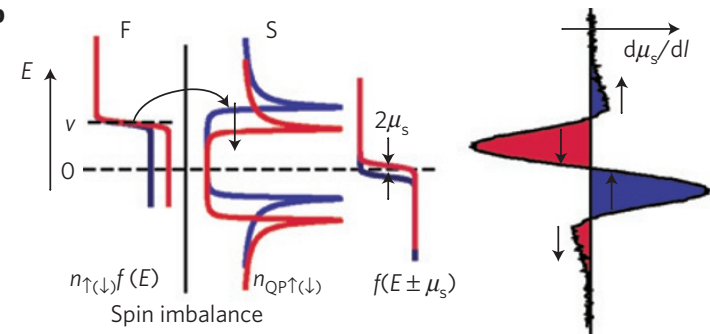

Figure $\mathbf{2}$ | Spin imbalance. (Device 8B3) a, Differential nonlocal resistance as a function of local voltage at different magnetic fields from $-1,418 \mathrm{G}$ (red) to $0 \mathrm{G}$ (blue). Anti-symmetric peaks due to spin imbalance are seen on a field-independent symmetric background due to charge imbalance (Inset). Peak height as a function of magnetic field (from anti-symmetrized data, Fig. 3). The straight line is a guide to the eye. b, A schematic representation of the theoretical model showing densities of states and distribution functions of various populations in $\mathrm{F} 1$ and $\mathrm{S}$. Owing to both the polarization of the ferromagnet and the Zeeman-split density of states in the superconductor, there is net spin accumulation in the superconductor and a shift in the chemical potentials of spin-up and spin-down quasiparticles. We measure the chemical potential of the spin-down and spin-up quasiparticles with respect to the Cooper pair condensate chemical potential.

occupation of all states is described by the Fermi-Dirac distribution function $f(E)=1 /\left(\mathrm{e}^{\beta E}+1\right)$, where $\beta=1 / k_{\mathrm{B}} T$ with $k_{\mathrm{B}}$ Boltzmann's constant and $T$ the temperature.

As discussed above, applying a current $I$ (and voltage $V$ ) between $\mathrm{F} 1$ and $\mathrm{S}$ gives rise to a finite spin accumulation in $\mathrm{S}$ and shifts the chemical potentials of spin-up and spin-down quasiparticles in the superconductor so that $f_{\mathrm{QP} \downarrow(\uparrow)}(E)=f\left(E \pm \mu_{\mathrm{S}}\right)$ (Fig. 2b).

Assuming $\mu_{\mathrm{S}} \ll \mathrm{eV}$, and using Fermi's golden rule ${ }^{13}$, we obtain for the spin current:

$$
\begin{aligned}
I_{\mathrm{S}}= & I_{\uparrow}-I_{\downarrow}=A M^{2} N_{\mathrm{N}} N_{\mathrm{F}}\left\{\frac{1+P}{2} \int n_{\mathrm{QP} \uparrow}(E)[f(E)-f(E+V)] \mathrm{d} E\right. \\
& \left.-\frac{1-P}{2} \int n_{\mathrm{QP} \downarrow}(E)[f(E)-f(E+V)] \mathrm{d} E\right\}
\end{aligned}
$$

Here $N_{\mathrm{N}}$ is the normal aluminium DOS at the Fermi level, $M$ the tunnelling matrix element (assumed to be constant in $E$ ) and $A$ a constant. The total current $I=I_{\uparrow}+I_{\downarrow}$ is given by a similar expression. 
a

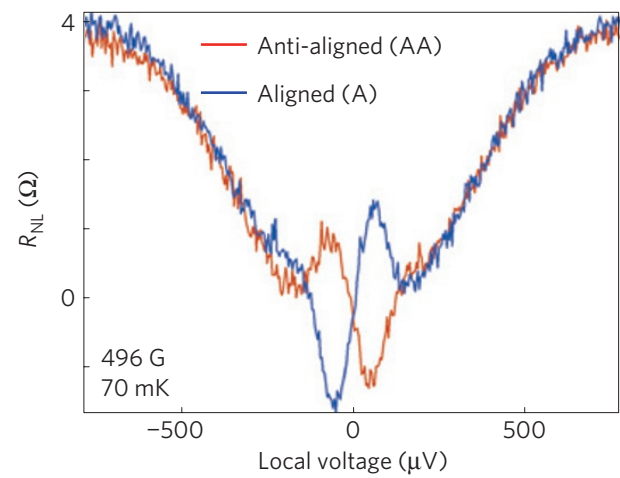

c

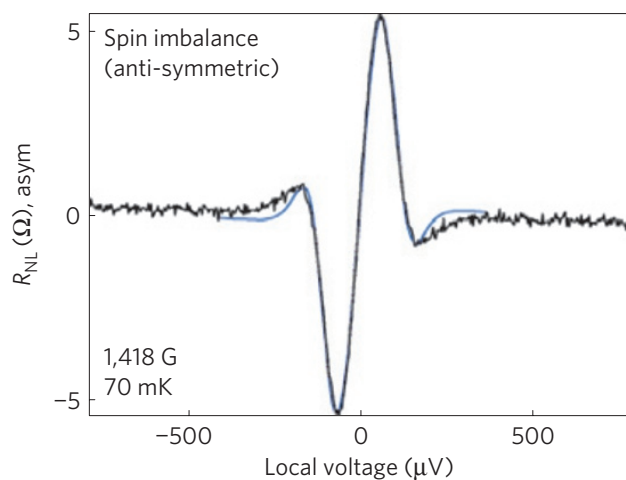

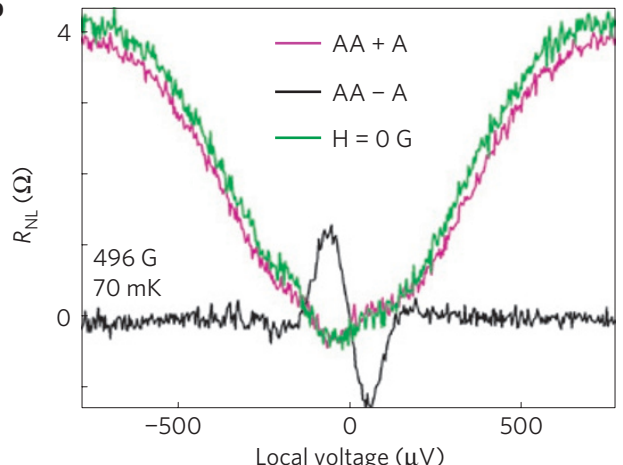

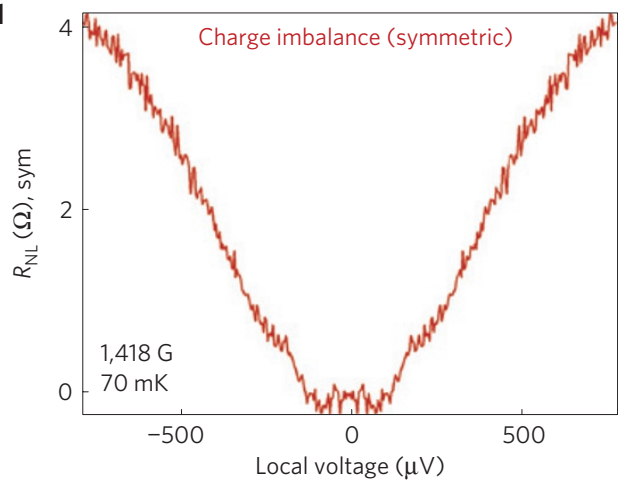

Figure 3 | Spin versus charge imbalance. (Device 8B3) a, Differential nonlocal resistance as a function of local voltage at $496 \mathrm{G}$ with the detector electrode aligned (blue line) then anti-aligned (red line) with the injector electrode and the magnetic field. The spin imbalance signal changes sign whereas the charge imbalance signal remains the same. The difference in amplitudes between the two spin signals is due to a residual magnetic field. (See Supplementary Information.) b. The sum and difference between the two traces (divided by two), giving approximately the charge and spin signals respectively. Note that the sum is almost identical to a trace taken at zero applied field. The effect of the residual field can be seen here. $\mathbf{c}$, The anti-symmetric part of the trace at $1,418 \mathrm{G}$ from Fig. 2a, due primarily to spin imbalance. The blue line is a fit to our theory, yielding a spin relaxation time of about $25 \mathrm{~ns}$. $\mathbf{d}$, The symmetric part of the trace at 1,418 G from Fig. $2 \mathrm{a}$, due primarily to charge imbalance.

The voltage drop detected at $\mathrm{J} 2$ due to the spin imbalance is refs 1,17 :

$$
\mu_{\mathrm{S}}=\frac{P_{\mathrm{d}} S^{*}}{2 N_{\mathrm{N}} g_{\mathrm{NS}} e}=\frac{P_{\mathrm{d}} I_{\mathrm{S}} \tau_{\mathrm{S}}}{2 N_{\mathrm{N}} g_{\mathrm{NS}} e^{2} \Omega}
$$

where $P_{\mathrm{d}}$ is the detector polarization, $\tau_{\mathrm{S}}$ the spin relaxation time, $e$ the electron charge, $\Omega$ the injection volume (the volume of the Al strip beneath the injection electrode) and $g_{\mathrm{NS}}$ the normalized detection junction conductance. We measure the spin differential resistance, $R_{\mathrm{S}}(V)=\mathrm{d} \mu_{\mathrm{S}} / \mathrm{d} I$ (Fig. $2 \mathrm{a}$ ).

Assuming further that $k_{\mathrm{B}} T, \mu_{\mathrm{B}} H \ll V$, we can expand in $\mu_{\mathrm{B}} H$ and replace the Fermi-Dirac functions with step functions to obtain

$$
R_{\mathrm{S}}(V)=\frac{P_{\mathrm{d}} \tau_{\mathrm{S}}}{2 N_{\mathrm{N}} g_{\mathrm{NS}} e^{2} \Omega}\left(P_{\mathrm{i}}+\frac{\mathrm{d} n_{\mathrm{QP}}(E) /\left.\mathrm{d} E\right|_{E=V}}{n_{\mathrm{QP}}(V)} \mu_{\mathrm{B}} H\right)
$$

where $P_{i}$ is the injector polarization and $n_{\mathrm{QP}}(E)$ is the quasiparticle density of states at zero field. (This helpful approximation is not made in the theoretical fits presented here. It makes little difference to the numerical results; see Supplementary Information.)

This expression is particularly suggestive: the spin imbalance can be seen here to depend clearly on the polarization of the injector electrode (first term) $)^{5,25}$ and on the Zeeman splitting of the DOS in the superconductor (second term) ${ }^{26,27}$. Therefore, in the presence of a magnetic field the injection electrode does not need to be polarized to create a spin imbalance; in principle a non-magnetic electrode would also work. From equation (1), we expect $R_{S}(V)$ to have a constant component (first term) and a component anti-symmetric in $V$ which grows linearly with magnetic field (second term). This is precisely what we observe in Fig. 2a, on a parabolic background.

To extract the spin signal, we note that $R_{\mathrm{S}}(V)$ is proportional to $P_{\mathrm{d}}$. In other words, if $P_{\mathrm{d}}$ changes sign then $R_{\mathrm{S}}(V)$ should do the same, whereas non-spin signals should remain unchanged. Therefore, in Fig. 3a, we measure $R_{\mathrm{S}}(V)$ with the detector oriented first one way (blue) then the other (red) at the same magnetic field. (A slight difference in amplitude is due to residual magnetic fields; see Fig. 3b and Supplementary Information.) Indeed, part of the signal changes sign whereas the parabolic background remains constant. We note that the sign-reversing part of the signal is essentially odd in $V$, with no constant offset (the red and blue curves cross at zero); this means that our spin signal comes primarily from the Zeeman-induced term above.

We can understand the dominance of the Zeeman-induced spin imbalance over the polarization-induced imbalance if they relax via different mechanisms - respectively elastic and inelastic - and thus over different timescales. In this case, we can write:

$$
R_{\mathrm{S}}(V)=\frac{P_{\mathrm{d}}}{2 N_{\mathrm{N}} g_{\mathrm{NS}} e^{2} \Omega}\left(P_{i} \tau_{\mathrm{S} 2}+\frac{\mathrm{d} n_{\mathrm{QP}}(E) /\left.\mathrm{d} E\right|_{E=V}}{n_{\mathrm{QP}}(V)} \mu_{\mathrm{B}} H \tau_{\mathrm{S} 1}\right)
$$

with $\tau_{\mathrm{S} 1} \gg \tau_{\mathrm{S} 2}$ at low temperature. The fact that the normal state magnetoresistance (Fig. 1c) is much smaller than the lowtemperature Zeeman-induced spin signal is consistent with this interpretation and suggests a relatively temperature-independent $\tau_{\mathrm{S} 2}$.

We now turn to the charge imbalance signal, which we can distinguish from the spin imbalance signal through symmetry considerations: $R_{\mathrm{C}}(V)=\mathrm{d} \mu_{\mathrm{C}} / \mathrm{d} I$ is an even function of $V$ (and of 

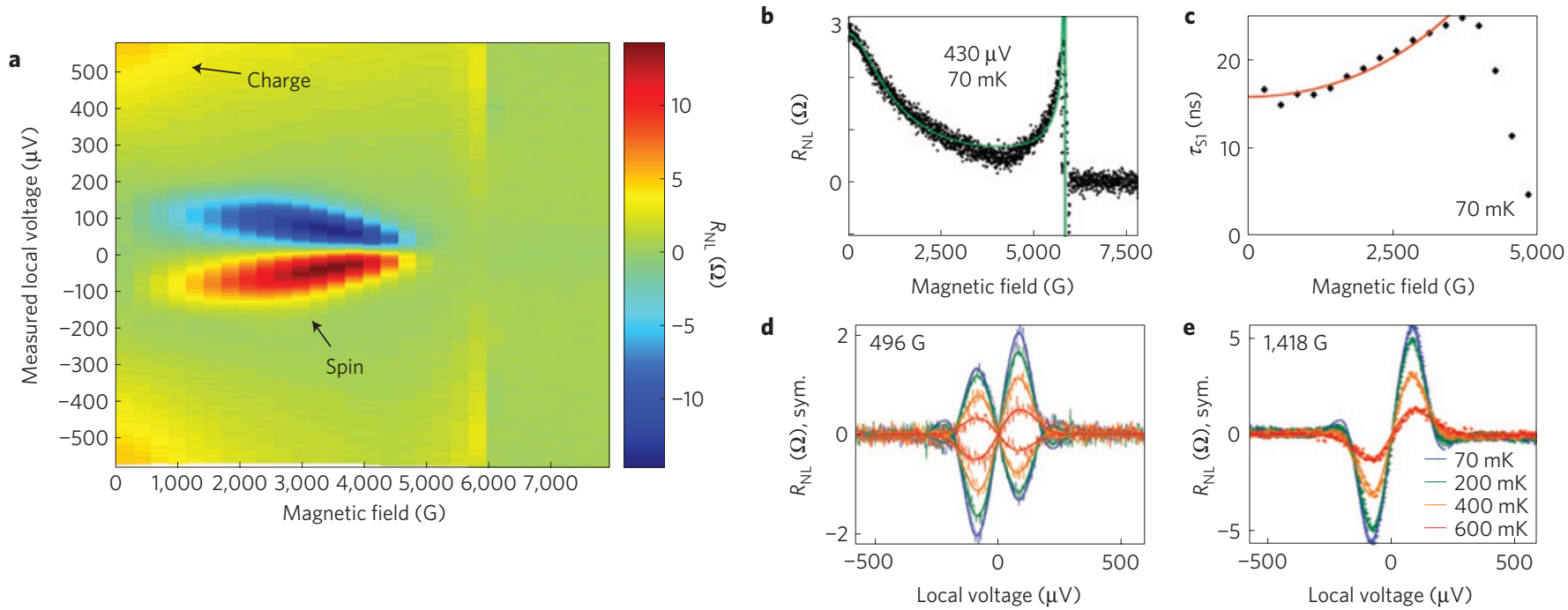

Figure 4 | High magnetic fields and temperature depedence. (Device 15A4) a, Differential nonlocal resistance as a function of local voltage and magnetic field up to the critical magnetic field and beyond. The disappearance of superconductivity at a critical field of about $6 \mathrm{kG}$ can be clearly observed. $\mathbf{b}$, A horizontal slice of $\mathbf{a}$ at $430 \mu \mathrm{V}$. The green line is a fit to theory, following ref. 31, yielding a charge relaxation time of $3 \pm 1 \mathrm{ps}$. $\mathbf{c}$, Estimated spin flip times obtained for fits to data in $\mathbf{a}$. The solid line is a fit to $\tau_{\mathrm{S} 1} \sim \sinh \left(g \mu_{\mathrm{B}} H / \mathrm{k}_{\mathrm{B}} T_{\text {eff }}\right) /\left(g \mu_{\mathrm{B}} H / \mathrm{k}_{\mathrm{B}} T_{\text {eff }}\right)$ with $T_{\text {eff }}=300 \mathrm{mK}$. This could be interpreted as a higher effective temperature for the quasiparticles. $\mathbf{d}, \mathbf{e}$, Temperature dependence of the spin imbalance signal. In $\mathbf{d}$, data are shown similar to those in Fig. $3 a$ (here anti-symmetrized) at different temperatures, and in $\mathbf{e}$, data are similar to those in Fig. 3c (legend in $\mathbf{e}$ also applies to $\mathbf{d}$ ). All traces, except for the one at $70 \mathrm{mK}$, are normalized by the relative detector $g_{\mathrm{NS}}$ (with respect to $70 \mathrm{mK}$; refs 1,2 ). Solid lines are theoretical fits.

$H$ ), whereas the Zeeman-induced $R_{S}(V)$ is odd in $V$ (and in $H$ ). (We have seen that the polarization-induced $R_{\mathrm{S}}(V)$, even in $V$ and $H$, is negligible.) This means that, for $R_{\mathrm{NL}}(V)=R_{\mathrm{C}}(V)+R_{\mathrm{S}}(V)$, the (anti-)symmetric component corresponds to the (spin) charge imbalance signal. Figure $3 \mathrm{c}, \mathrm{d}$ show these signals separately for the $B=1,418 \mathrm{G}$ trace in Fig. 2a.

We see here that the spin signal is maximal at a voltage at which the charge signal is negligibly small, and that conversely the charge imbalance becomes non-negligible at higher voltages where there is no spin imbalance. Spin and charge signals are thus well separated in energy.

To obtain the spin relaxation time $\tau_{\mathrm{S} 1}$, we fit our theory to the spin signal (Fig. 3c, blue line) with $\tau_{\mathrm{S} 1}$ as the only free parameter. This yields $\tau_{\mathrm{S} 1} \sim 25 \mathrm{~ns}$. (The DOS used in the fit is that measured across J1.) Furthermore, the magnetic-field dependence of the spin relaxation time (Fig. 4c) $\tau_{\mathrm{S} 1} \sim \sinh \left(g \mu_{\mathrm{B}} H / k_{\mathrm{B}} T_{\text {eff }}\right) /\left(g \mu_{\mathrm{B}} H / k_{\mathrm{B}} T_{\text {eff }}\right)$ for fields less than about half the critical field confirms that spin relaxation occurs primarily through inelastic scattering processes ${ }^{28}$, which are 'frozen out' by the magnetic field. (Fig. 4b)

To compare $\tau_{\mathrm{S} 1}$ to the charge relaxation time $\tau_{\mathrm{Q}}$, we measure the charge imbalance signal at high bias voltage $(V=430 \mu \mathrm{V})$ as the magnetic field is increased. As pointed out in ref. 29, there is a one-to-one correspondence between (field-induced) depairing and charge relaxation. Therefore, $R_{\mathrm{C}}$ initially decreases owing to the field-induced pair-breaking ${ }^{30}$, then diverges at the critical field $H_{\mathrm{C}} \sim 6 \mathrm{kG}$ as the superconducting gap goes to zero, before dropping abruptly to zero in the normal state ${ }^{31}$. A theoretical fit (Fig. 4b, green line, see Supplementary Section SB for details) yields a charge relaxation time of $\tau_{\mathrm{Q}}=3 \pm 1 \mathrm{ps} \ll \tau_{\mathrm{S} 1}$.

Figure $4 \mathrm{a}$ shows the nonlocal resistance as a function of local voltage over a larger range of magnetic fields and summarizes our main results: the asymmetric (red and blue) spin signal grows with magnetic field then diminishes and becomes narrower in $V$ as the superconducting gap decreases. In the background, the charge signal decreases, then diverges with magnetic field. Both disappear at the critical field.

We also investigate the temperature evolution of the spin imbalance signal at $B=296 \mathrm{G}$ for both aligned and anti-aligned states
(Fig. $4 \mathrm{~d}$ ) and at $B=1,418 \mathrm{G}$ (Fig. 4e). The magnitude of the spin signal diminishes with increasing temperature, owing primarily to temperature broadening of all distribution functions in the system. Theoretical fits to the data in Fig. $4 \mathrm{e}$ tell us that $\tau_{\mathrm{S} 1}$ decreases with increasing temperature: $\tau_{\mathrm{S} 1}=14.2,14.1,12.3,7.9 \mathrm{~ns}$ at $T=70$, 200,400 and $600 \mathrm{mK}$ respectively ${ }^{32}$. This temperature dependence is consistent with theoretical predictions of spin-lattice relaxation times of conduction electrons in the superconducting state ${ }^{33}$.

After submitting our manuscript we became aware of similar work, described in ref. 34 .

\section{Methods}

We fabricated our samples with standard electron-beam lithography and angle evaporation techniques in an electron-beam evaporator with a base pressure of $5 \times 10^{-9} \mathrm{mbar}$. We first evaporate $20 \mathrm{~nm}$ of $\mathrm{Al}$, which is then oxidized at $10^{-2} \mathrm{mbar}$ for $10 \mathrm{~min}$ to produce a tunnel barrier, then $50 \mathrm{~nm}$ of Co and finally $20 \mathrm{~nm}$ of Pd as a capping layer. The Pd capping layer reduces the overall device resistance; prevents oxidation of the Co; and smooths out magnetic textures in the Co. All transport measurements were done in a ${ }^{3} \mathrm{He}-{ }^{4} \mathrm{He}$ dilution refrigerator with a base temperature of $70 \mathrm{mK}$. The a.c. excitation current was modulated at $37 \mathrm{~Hz}$; its amplitude was $1 \mu \mathrm{A}$ at $4 \mathrm{~K}$ and $10 \mathrm{nA}$ at all other temperatures. Sample fabrication and measurement circuit details are provided in Supplementary Sections SC and SD.

Received 28 July 2012; accepted 27 November 2012; published online 13 January 2013

\section{References}

1. Clarke, J. Experimental observation of pair-quasiparticle potential difference in nonequilibrium superconductors. Phys. Rev. Lett. 28, 1363-1366 (1972).

2. Tinkham, M. \& Clarke, J. Theory of pair-quasiparticle potential difference in nonequilibrium superconductors. Phys. Rev. Lett. 28, 1366-1369 (1972).

3. Aronov, A. Spin injection and polarization of excitations and nuclei in superconductors. J. Exp. Theor. Phys. 44, 193-196 (1976).

4. Johnson, M. Spin coupled resistance observed in ferromagnet-superconductor-ferromagnet trilayers. Appl. Phys. Lett. 65, 1460-1462 (1994).

5. Takahashi, S., Imamura, H. \& Maekawa, S. Spin imbalance and magnetoresistance in ferromagnet/superconductor/ferromagnet double tunnel junctions. Phys. Rev. Lett. 82, 3911-3914 (1999).

6. Chen, C. D., Kuo, W., Chung, D. S., Shyu, J. H. \& Wu, C. S. Evidence for suppression of superconductivity by spin imbalance in Co-Al-Co single-electron transistors. Phys. Rev. Lett. 88, 047004 (2002). 
7. Tinkham, M. Tunneling generation, relaxation, and tunneling detection of hole-electron imbalance in superconductors. Phys. Rev. B 6, 1747-1756 (1972)

8. Schmid, A. \& Schön, G. Linearized kinetic equations and relaxation processes of a superconductor near $T_{C}$. J. Low Temp. Phys. 20, 207-227 (1975).

9. Clarke, J. in Nonequilibrium Superconductivity, Phonons, and Kapitza Boundaries (ed. Grey, K. E.) 353-422 (NATO Science Series B, Vol. 65, Springer, 1981).

10. Leridon, B., Lesueur, J. \& Aprili, M. Spin-bottleneck due to spin-charge separation in a superconductor. Phys. Rev. B 72, 180505 (2005).

11. Cadden-Zimansky, P., Jiang, Z. \& Chandrasekhar, V. Charge imbalance, crossed Andreev reflection and elastic co-tunnelling in ferromagnet/superconductor/normal-metal structures. New J. Phys. 9, 116 (2007).

12. Kivelson, S. A. \& Rokhsar, D. S. Bogoliubov quasiparticles, spinons, and spin-charge decoupling in superconductors. Phys. Rev. B 41, 11693-11696 (1990)

13. Zhao, H. L. \& Hershfield, S. Tunneling, relaxation of spin-polarized quasiparticles, and spin-charge separation in superconductors. Phys. Rev. B 52 , 3632-3638 (1995).

14. Tinkham, M. Introduction to Superconductivity 2nd edn (Dover, 2004).

15. Chiodi, F., Aprili, M. \& Reulet, B. Evidence for two time scales in long SNS junctions. Phys. Rev. Lett. 103, 177002 (2009)

16. Jedema, F. J., Heersche, H. B., Filip, A. T., Baselmans, J. J. A. \& van Wees, B. J. Electrical detection of spin precession in a metallic mesoscopic spin valve. Nature 416, 713-716 (2002).

17. Johnson, M. \& Silsbee, R. H. Spin-injection experiment. Phys. Rev. B 37, 5326-5335 (1988).

18. Valenzuela, S. O. \& Tinkham, M. Spin-polarized tunneling in room-temperature mesoscopic spin valves. Appl. Phys. Lett. 85, 5914-5916 (2004).

19. Münzenberg, M. \& Moodera, J. S. Superconductor-ferromagnet tunneling measurements indicate sp-spin and d-spin currents. Phys. Rev. B 70, 060402 (2004)

20. Alvarado, S. F. Tunneling potential barrier dependence of electron spin polarization. Phys. Rev. Lett. 75, 513-516 (1995).

21. Tedrow, P. M. \& Meservey, R. Spin polarization of electrons tunneling from films of Fe, Co, Ni, and Gd. Phys. Rev. B 7, 318-326 (1973).

22. Meservey, R. \& Tedrow, P. Spin-polarized electron tunneling. Phys. Rep. 238, 173-243 (1994).

23. Fulde, P. High field superconductivity in thin films. Adv. Phys. 22, 667-719 (1973).

24. Anthore, A., Pothier, H. \& Esteve, D. Density of states in a superconductor carrying a supercurrent. Phys. Rev. Lett. 90, 127001 (2003).

25. Poli, N. et al. Spin injection and relaxation in a mesoscopic superconductor. Phys. Rev. Lett. 100, 136601 (2008).
26. Huertas-Hernando, D., Nazarov, Y. V. \& Belzig, W. Absolute spin-valve effect with superconducting proximity structures. Phys. Rev. Lett. 88, 047003 (2002).

27. Bergmann, G., Lu, J. \& Wang, D. Meservey-Tedrow effect in ferromagnet/superconductor/ferromagnet double tunnel junctions. Phys. Rev. B 71, 134521 (2005).

28. Pierre, F. et al. Dephasing of electrons in mesoscopic metal wires. Phys. Rev. B 68, 085413 (2003).

29. Lemberger, T. R. One-to-one correspondence of charge-imbalance relaxing mechanisms with pair-breaking mechanisms in superconductors. Phys. Rev. B 29, 4946-4950 (1984).

30. Hübler, F., Lemyre, J. C., Beckmann, D. \& v Löhneysen, H. Charge imbalance in superconductors in the low-temperature limit. Phys. Rev. B 81, 184524 (2010).

31. Kleine, A. et al. Magnetic field and contact resistance dependence of non-local charge imbalance. Nanotechnology 21, 274002 (2010).

32. Yang, H., Yang, S., Takahashi, S., Maekawa, S. \& Parkin, S. S. P. Extremely long quasiparticle spin lifetimes in superconducting aluminium using $\mathrm{MgO}$ tunnel spin injectors. Nature Mater. 9, 586-593 (2010).

33. Yafet, Y. Conduction electron spin relaxation in the superconducting state. Phys. Lett. A 98, 287-290 (1983).

34. Hübler, F., Wolf, M. J., Beckmann, D. \& v. Löhneysen, H. Long-range spin-polarized quasiparticle transport in mesoscopic Al superconductors with a Zeeman splitting. Phys. Rev. Lett. 109, 207001 (2012).

\section{Acknowledgements}

We thank C. Strunk, B. Reulet, J. Gabelli, B. Leridon, Y. Nazarov, D. Beckmann and J. Lesueur for discussions on spin injection; S. Rohart for advice on magnetic materials; S. Autier-Laurent for technical assistance; and S. Guéron, J. Gabelli and R. W. Ogburn for comments on the manuscript. This work was funded by a European Research Council Starting Independent Researcher Grant (NANO-GRAPHENE 256965), a C'NANO grant (DYNAH) from the Ile-de-France region and an ANR Blanc grant (DYCOSMA) from the French Agence Nationale de Recherche.

\section{Author contributions}

C.Q.H.L. and M.A. fabricated the samples and performed the measurements. All the authors contributed to the data analysis and the writing of the manuscript.

\section{Additional information}

Supplementary information is available in the online version of the paper. Reprints and permissions information is available online at www.nature.com/reprints. Correspondence and requests for materials should be addressed to C.Q.H.L.

\section{Competing financial interests}

The authors declare no competing financial interests. 